\title{
Avaliação da qualidade de assistência primária à saúde em localidade urbana da região sul do Brasil
}

\author{
Quality evaluation of primary health care an urban area of southern Brazil
}

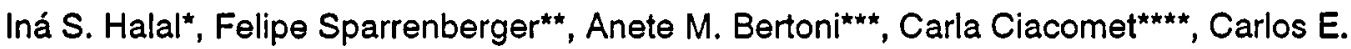

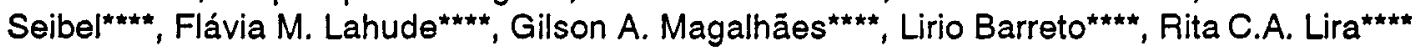

\begin{abstract}
HALAL, I.S. et al. Avaliação da qualidade de assistência primária à saúde em localidade urbana da região sul do Birsil. Rev. Saíde Pública, 28: 131-6,1994. Foi estudada transversalmente uma amostra de $10 \%$ dos pacientes que consultaram em dois Postos de Assistência Primária à Saúde da Cidade de Pelotas (RS), Brasil, com o objctivo de avaliar, qualitativamente, o cuidado à saúde que estava sendo oferecido. Através de entrevistas domiciliares, 15 dias após a consulta, a resolução do problema (cura ou melhora) foi alcançada em $87,9 \%$ dos pacientes. A satisfação do paciente ou de seu responsável, no caso de consultas pediátricas, foi observada em cerca de $90 \%$ dos casos e esteve associada estatisticamente com a resolução do problema ( $p=0,04)$. Observoul-se associação entre resolutividade e disponibilidade de medicamentos no Posto. Os pacientes que receberam todo ou pelo menos parte do tratamento tiveram uma probabilidade $33 \%$ maior de terem seu problema resolvido. A satisfação dos profissionais mostrou-se linearmente associada com a percepção de melhor relação profissional-paciente ( $R P=3,48$; IC 95\% 2,17 - 5,59) e com a expectativa de melhor prognóstico para o paciente $(R P=1,99 ; \mathrm{IC} 95 \% 1,36-2,91)$.
\end{abstract}

Descritores: Cuidados primários de saúde. Avaliação de processos e resultados (cuidados de saúde). Satisfação do paciente.

\section{Introdução}

Inúmeros estudos têm sido realizados ${ }^{1,4,10}$, em diferentes países, com o objetivo de avaliar a qualidade dos serviços de saúde, dos ponto de vista dos pacientes. No Brasil existem poucos estudos de avaliação de serviços de saúde. A pequena produção se restringe praticamente à avaliação quantitativa dos serviços ${ }^{2}$, restando escassas informações sobre resolutividade e satisfação dos pacientes. Segundo Donabedian ${ }^{3}$, a avaliação qualitativa dos serviços comporta sempre duas dimensões: desempenho técnico, ou seja, aplicação do conhecimento e da tecnologia médica, de modo a maximizar os beneficios e reduzir os riscos; e relacionamento com o paciente. A avaliação qualitativa do cuidado à saúde, segundo o citado autor, pode ser realizada em seus três componentes: estrutura, pro-

- Departamento de Medicina Social da Universidade Federal de Pelotas (UFPel) - Pelotas, RS - Brasil

* Residência de Medicina Preventiva e Social da UFPel . Pelotas, RS - Brasil

-* FAU da UFPel - Pelotas, RS - Brasil

Acadêmicos de Medicina da UFPel Pelotas, RS - Brasil

Separatas/Reprints: I.S.Halal - Av. Duque de Caxias, 250 . Fragata- Caixa Postal 464 - 96030-000. Pelotas, RS - Brasil cesso e resultado. A estrutura refere-se às características dos recursos utilizados pelo serviço; o processo, aos procedimentos empregados no manejo dos problemas apresentados pelos pacicntes; e, o resultado, ao estado de saúde do paciente ou da comunidade resultante da interação com o scrviço de saúde. A satisfação do paciente, o objctivo mais importante nos estudos cle avaliação de qualidade ${ }^{3}$, tem sido inclúda, geralmente, na avaliação de resultados. $O$ citado autor inclui a satisfação dos profissionais de saúde como importante elemento da qualidade dos serviços oferecidos à população. A qualidacle do cuidado oferecido, portanto, é multifatorial ${ }^{7}$. Desta forma, o presente estudo visa a avaliar qualitativamente a capacidade resolutiva e a satisfação em uma amostra de pacientes e profissionais da rede de Assistência Primária à Saúde da Cidade de Pelotas (RS).

\section{Material e Método}

Os dados aqui apresentados foram obtidos através da realização de um estudo transversal em dois postos de saúde da rede de assistência primária à saúde da Cidade de Pelotas (RS). A área de abrangência dos dois postos cobre uma 
população em torno de 8.500 pessoas. Esses postos são gerenciados e financiados pela Universidade Federal de Pelotas, e a demanda mensal é de cerca de 2.500 consultas. Os serviços prestados à população pelas equipes de saúde diversificam-se desde procedimentos preventivos, preponderantemente na área da saúde materno-infantil (vacinas, monitorização do crescimento e desenvolvimento da criança, pré-natal, prevenção do câncer ginecológico) até o alcndimento de pacientes com problcmas agudos ou crônicos. Ambos os postos estão estruturados com equipes multiprofissionais de saúde e funcionam como postos-escola para médicos residentes e estudantes de graduação de vários cursos da área da saúde da Universidade Federal.

O objetivo do presente estudo foi o de avaliar o impacto sobre a resolutividadc e sobre a satisfação dos pacientes e dos profissionais das seguintes variáveis: tipo de problema; prognóstico do paciente, considerando fatores biopsicossociais; prescrição de medicamentos e adesão ao tratamento; solicitação de exames complementares e sua realização; cncaminhamento para outros profissionais e garantia de acesso; e, qualidade da relação profissional-paciente. Entende-se por resolutividade a capacidade do serviço de solucionar os problemas que lhe são apresentados; e, por satisfação, a percepção subjetiva de pacientes e profissionais quanto ao alcance de suas expectativas.

Para a avaliação da resolutividade e da satisfação do usuário, utilizou-se um processo sistemático de amostragem, de forma a obter-se informação de $10 \%$ dos pacientes que tiveram consulta em cada um dos postos, no período de 23.10.92 a 16.11.92. Os pacientes eram entrevistados no periodo máximo de 15 dias após a realização da consulta. Para a avaliação da satisfação dos profissionais foram entrevistados todos os profissionais de nível superior ao final de um turno de trabalho ( 4 horas), no período de 16.11.92 a 04.12.92.

Para a avaliação do grau de resolução de problemas e de satisfação dos usuários foram utilizados questionários especialmente delincados e pré-testados. Esses foram aplicados aos pacientes (ou aos pais dos pacientes, para o caso de consultas pediátricas) em entrevistas domiciliares por entrevistadores treinados. Os dados de identificação e endereço do paciente foram obticlos através dos registros dos postos. Para a avaliação da satisfação dos profissionais de saúcle utilizou-se questionário auto-explicativo e auto-aplicável que cra preenchido na presença de um membro da equipe de pesquisa. A satisfação do profissional foi definicla através de uma escala demarcada de um a dez em que o profissional escolhia, visualmente, o cscore de satisfação que melhor correspondia a cada consulta.

$\Lambda$ pós a revisão do conteúdo e da codificação dos questionários, os dados foram digitados $\mathrm{cm}$ microcomputador, utilizando-se o programa Epi Info'. As análises foram feitas com o programa SPSS/PC+ ${ }^{8}$. O teste qui-quadrado foi usado para avaliar a lorça das associações, e o risco relativo foi calculado através das razões de prevalências.

\section{Resultados}

Cento e dezenove pacientes deveriam compor a amostra estudada, perfazendo $10 \%$ da demanda atendida nos 15 dias que precediam a entrevista. Desses, conscguiu-se localizar $^{99}(83 \%)$. Dos 20 pacientes que não foram encontrados, 9 residiam fora de área e haviam tido consulta no scrviço informando endereço de pessoa da família cadastrada no Posto, 7 haviam mudado de endereço $\mathrm{c}$ a vizinhança não dispunha do endereço atual, 3 não foram encontrados após três visitas em horários e dias diferentes e um paciente havia viajado para outra cidade. $\Lambda$ amostra ficou então constiluída por 128 pessoas, sendo 99 pacientes que foram entrevistados $\mathrm{cm}$ scu domicilio e 29 profissionais de saúcle que executavam suas funções nos dois postos periféricos estudados.

\section{Resolução do problema e satisfação dos pacientes}

Quanto às características socioeconômicas e demográficas da populaçĩo estudada, $46 \%$ dos pacientes eram crianças e adolescentes (menores de 20 anos), $43 \%$ eram adultos e $11 \%$ pertenciam ao grupo de 60 anos ou mais de idade. A média de escolaridade das pessoas entrevistadas foi de 3,7 anos (desvio-padrão de 2,9 anos), sendo que $22 \%$ eram analfabetos. A renda familiar média foi de 2,1 salários-mínimos da época. 
Tabela 1. Distribuição de características das consultas em assistência primária à saúde. Pelotas, RS, 1992.

\begin{tabular}{lcc}
\hline Caracteristica & Percentagem & N \\
\hline Prescrição de medicamentos & 55,7 & 54 \\
Solicitação de exames complementares & 19,4 & 19 \\
Encaminhamento para outro profissional & 5,1 & 5 \\
\hline
\end{tabular}

Tabela 2. Resolução do problema e satisfação do paciente duas semanas após a consulta, de acordo com a opiniāo do paciente. Pelotas, RS, 1992.

\begin{tabular}{lcc}
\hline Opinião do paciente & Percentagem & $\mathrm{N}$ \\
\hline Situação do problema & & \\
Resolvido & 46,5 & 46 \\
Melhor & 35,3 & 35 \\
Igual & 16,2 & 16 \\
Pior & 2,0 & 2 \\
Satisfeito com a consulta & & \\
Sim & 87,9 & 87 \\
Nāo & 6,1 & 6 \\
Mais ou menos & 6,1 & 6 \\
\hline Total & 100 & 99 \\
\hline
\end{tabular}

A Tabela 1 mostra que $55,7 \%$ das consultas resultaram em prescrição de medicamentos. Foram solicitados exames complementares em $19,4 \%$ das consultas; e, apenas $5 \%$ dos pacientes foram encaminhados para outros profissionais. Quanto ao problema que motivou a consulta (Tabela 2), quinze dias após os pacientes ou seus responsáveis relataram que estavam me- lhor ou tinham resolvido o problema em $35,3 \%$ e $46,5 \%$ dos casos, respectivamente. Cerca de $88 \%$ dos pacientes ou seus responsáveis relataram estar satisfeitos com a consulta (Tabela 2 ), o que esteve significativamente associado com a resolução do problema $(p=0,04)$.

$\Lambda$ Tabcla 3 mostra o grau de resolução dos problemas scgundo a opinião do paciente, de acordo com a prescrição de medicamentos. A resolução ou a melhora do problema foi duas vezes mais freqücnte entre os pacientes que conseguiram fazer o tratamento prescrito $(p=0,00$; IC $95 \%$ de 1,27 a 4,25). Os pacientes que receberam toda ou parte da medicação no próprio posto Liveram uma probabilidacle de $33 \%$ maior de terem seu problema resolvido ou melhorado nos quinze dias subseqüentes à consulta $(p=0,004$; IC 95\% 1,01-1,75) quando comparados com os clemais. Poder comprar todo o tratamento também esteve associado com probabilidade maior de ter o problema resolvido. Quando comparados com os pacientes que não receberam medicação gratuita, os que puderam comprar todo o tratamento tiveram uma probabilidade $25 \%$ maior de melhorar ou de ter seu problema de saúde resolvido em igual periodo de quinze dias. Quando a medicação era reccitada para ser obtida total ou parcialmente na farmácia do Instituto Nacional de Seguriclade Social (INSS), a probabilidade de resolução do problema ficou reduzida em cerca de $40 \%(p=0,06$ IC 95\% 0,34-0,97).

As demais variáveis independentes estudadas não apresentaram associação estatisticamente significativa com resolução do problema ou satisfação do paciente.

Tabela 3. Grau de resolução de problemas em assistência primária à saúde conforme prescrição de medicamentos segundo a opiniäo dos pacientes, Pelotas, RS, 1992.

\begin{tabular}{|c|c|c|c|}
\hline Caracteristica da prescrição & $\mathrm{N}$ & $\begin{array}{l}\text { Problema resolvido ou melhora } \\
(\%)\end{array}$ & Razāo de prevalências (IC95\%) \\
\hline Tomou os medicamentos & $\operatorname{Sim} 51(86,4 \%)$ & $86,3 \%$ & $2,32(1,27-4,25)$ \\
\hline prescritos & Não $8(13,6 \%)$ & $37,5 \%$ & 1,00 \\
\hline Recebeu todo ou parte & $\operatorname{Sim} 24(38,1 \%)$ & $95,8 \%$ & $1,33(1,01-1,75)$ \\
\hline do tratamento no posto & Não $39(61,9 \%)$ & $71,8 \%$ & 1,00 \\
\hline \multicolumn{4}{|l|}{ Retirou todo ou parte } \\
\hline de tratamento na farmácia & $\operatorname{sim} 8(14,3 \%)$ & $50,0 \%$ & $0,57(0,34-0,97)$ \\
\hline da Central de Medicamentos & Não $48(85,7 \%)$ & $85,4 \%$ & 1,00 \\
\hline \multirow[t]{2}{*}{ Comprou todo o tratamento } & $\operatorname{Sim} 22(39,3 \%)$ & $90,9 \%$ & $1,25(1,14-1,77)$ \\
\hline & Năo $34(60,7 \%)$ & $73,5 \%$ & 1,00 \\
\hline
\end{tabular}




\section{Satisfação dos profissionais}

Foram estudadas as opiniões dos 29 profissionais de nivel superior que trabalhavam nos dois postos de saúde. Ao final de um turno de trabalho dos 29 profissionais, 109 pacientes haviam sido atendidos. Setenta e oito por cento das consultas foram realizadas por médicos, $15 \%$ por enfermeiras, $6 \%$ por nutricionistas e $1 \%$ por assistentes sociais. Atividades preventivas por excelência (puericultura e pré-natal) constitúram o principal motivo de atendimento em ambos os postos de saúde ( $16 \%$ das queixas principais e $13 \%$ dos diagnósticos). Em $67,3 \%$ das consultas os profissionais se sentiram muito satisfeitos (grau de satisfação 8 a 10, inclusive, na escala); $\mathrm{cm} \mathrm{28 \% ,}$ mediante satisfeitos (grau de satisfação 4 a 7); e, em apenas $4,7 \%$ deles se sentiram insatisfeitos. $O$ "score" médio de satisfação dos profisssionais foi de 7,8 (desvio-padrão 2,2).

A relação profissional-paciente, segundo a percepção dos primeiros, foi considerada "muito boa" em $58,7 \%$ das consultas; "boa" em $37,5 \%$ e "ruim" em apenas 3,8\% clelas (Tabcla 4). $\Lambda$ prevalência de satisfação do profissional aumentou de $25 \%$, quando a relação profìssional-paciente foi considerada "ruim", para cerca de $90 \%$ nos casos em que foi considerada "muito boa".

Em 56,7\% dos pacientes (Tabela 4), o prognóstico imediato esperaclo pelos profissionais, em função da intervenção da consulta, cra de "cura", isto é, resolução completa do problema. Os profissionais esperavam "melhora" do paciente em 26\% dos casos; impossibilidade de modificação ("igual") em 9,6\%; e, em 7,7\% das consultas, o prognóstico foi considerado "imprevisível". Em nenhum caso o prognóstico esperaclo foi de "piora" do paciente. A prevalência de satisfação do profissional aumentou com a melhora do prognóstico. Quando a cura era o prognóstico esperado, a prevalência de satisfação do profissional foi de cerca de $80 \%$, caindo para cerca de $40 \%$ quando não se esperava modificação do problema após a consulta ou quando a evolução do paciente fosse considerada imprevisivel.

Tanto a relação profissional-paciente, quanto o prognóstico csperado estiveram ambos significativamente associados com a probabilidade de satisfação do profissional $(\mathrm{p}=0,000$ e $\mathrm{p}=0,4$, respectivamentc). $\Lambda$ Tabela 4 mostra que nas consultas em que a relação profissionalpaciente foi considerada "muito boa", a probabilidade de satisfação do profissional foi 3,5 vezes maior do que no grupo $\mathrm{cm}$ que csta foi considerada "ruim". Obscrvou-se também tendência à lincaridade entre as duas variáveis $(p=$ $0,00)$, isto é, quanto melhor a relação percebida pelo profissional, maior a probabilidade de satisfação com a consulta. Quanto ao prognóstico, a probabilidade de satisfação foi cerca de duas vezes maior quando o profissional esperava resolução completa do problema. A possibilidade de melhora do paciente esteve associada com uma probabilidade de satisfação do profissional $40 \%$ maior do que nos casos $\mathrm{cm}$ que o prognóstico não cra passível de modificação com a consulta. O teste para tendência linear entre as duas variáveis foi significativo ao nível de $p=0,009$.

Tabela 4. Grau de satisfação dos profissionais da assistência primária à saúde conforme caracteristicas de consulta. Pelotas, RS, 1992.

\begin{tabular}{cccc}
\hline Caracteristica & $N$ & Satisfação do profissional & Razão de prevalèncias (IC95\%) \\
\hline $\begin{array}{c}\text { Relação profissional-paciente } \\
\text { Muito boa }\end{array}$ & & \\
Boa & $61(58,7 \%)$ & $86,9 \%$ & $3,48(2,17-5,59)$ \\
Ruim & $39(37,5 \%)$ & $43,2 \%$ & $1,64(1,36-1,98)$ \\
Prognóstico do problema & $4(3,8 \%)$ & $25,0 \%$ & 1,00 \\
Cura & $59(56,7 \%)$ & $79,7 \%$ & $1,99(1,36-2,91)$ \\
Melhora & $27(26,0 \%)$ & $56,0 \%$ & $1,40(1,16-1,68)$ \\
Igual & $10(9,6 \%)$ & $40,0 \%$ & 1,00 \\
Imprevisivel & $8(7,7 \%)$ & $37,5 \%$ & $0,94(0,91-0,97)$ \\
\hline
\end{tabular}




\section{Discussão}

A capacidade resolutiva de um serviço de saúde supõe dois aspectos ${ }^{2}$ a capacidade de absorver o número de assistência solicitada (capacidade resolutiva quantitativa ou acesso), e a capacidade de resolver os casos atendidos (capacidade resolutiva qualitativa). $\Lambda$ capacidade resolutiva qualitaliva, objeto do presente estudo, representa a capacidade do scrviço $\mathrm{em}$ solucionar a maior parte dos problemas que lhe são apresentados. Existem vários indicadores propostos para avaliação de resultados, desde medidas de morbidade até medidas de progesessão prevenível de doenças. A percepção própria do paciente também tem sido considcrada uma medida da resolutividade dos serviços ${ }^{3}$. Segundo a percepção dos pacientes, no presente trabalho, a resolutividade qualitativa alcançada, 15 dias após o contato com o scrviço de saúde, foi de $87,9 \%$. Entre as variáveis independentes estudadas, a resolução do problema foi dependente apenas da clisponibilidade de medicamentos. Poder lazer o tratamento prescrito correspondeu a uma probabilidade duas vezes maior de resolução do problcma, no espaço de 15 dias. Em termos de disponibilidade de medicamentos, receber os rcmédios no próprio posto de saúde esteve associado com uma probabilidade $33 \%$ maior de resolução do problema quando comparados com os demais pacientes. As demais variáveis independentes estudadas não apresentaram associação estatisticamente significativa com a resolutividade, provavelmente em função de dois aspectos: estratos muito pequenos em cada uma das categorias da variável, e uniformidade da amostra quanto a fatores sociais importantes na determinação do processo saúde-doença, como renda e escolaridade.

Cerca de $90 \%$ dos pacientes, ou seus responsáveis, se disscram satisfeitos com o atendimento recebido no Posto. Em geral, os pacientes mostram-se relutantes em expressar sua não satisfação com os serviços de saúde2,10. Alćm disso, vários fatores podem afetar a percepção dos pacientes, desde experiências prévias até o estado atual de saúde. No presente estudo, a satisfação com a consulta esteve significativamente associada com a resolução do problema $(p=0,04)$, segundo a percepção do próprio paciente ou de scu responsável, o que reforça a possibiliclacle de real satisfação. Não foram utilizados outros marcadores que pudessem validar a informação de resolução do problema ou de satislaçĩo do paciente. $\Lambda$ s perguntas que forneciam cstas informações, no entanto, foram colocadas estratcgicamente ao final da entrevista, depois de se ter garantido um clima de confiança entre o cntrevistador e o entrevistado.

$\Lambda$ satisfação do profissional tem sido sugericla como uma dimensão importante da avaliação da çualidade dos scrviços ${ }^{3}$. No presente trabalho, os 29 profissionais de nivel superior, trabalhando $\mathrm{cm}$ assistência primária à saúde, revelaram-se muito satisfeitos em $67,3 \%$ das consultas. A qualidade da relação profissionalpacientc, de acordo com a percepção dos primciros, foi importante fator associado com a satislação profissional. Embora não se tenha utilizaclo marcadores de validação da percepção do prolissional, as consultas em que a relação profissional-paciente foi considerada "muito boa" tiveram prevalência de satisfação por parte do profissional de cerca de $90 \%$. A probabilidacle de satisfação prolisssional no atendimento de pacientes foi 3,5 vezes maior quando a relação cstabelecida entre ambos foi considerada "muito boa".

$\Lambda$ incerteza prognóstica ("prognóstico imprevisivel") ou a incapaciclade de alterar o curso da docnça do paciente ("prognóstico igual") estiveram associados com a menor prevalência de satislação profissional (cerca de 40\%). Mesmo que a resolução completa do problema não pudesse ser esperada, em função da cronicidade da doença ou de seu aspecto recidivante ou de fatores sociais adversos, o prognóstico de melhora do paciente esteve associado com uma prevalência de cerca de $60 \%$ de satisfação profissional. O manejo de situações cuja expectativa fosse a completa resolução apresentaram uma probabilidade duas vezes maior de satisfação por parte do profissional.

Mesmo considerando que a maior concentração de motivos de consultas e de diagnósticos dos profissionais ocorreu em atividades preventivas (puericultura e pré-natal) o que, por si só, possivelmente se relacione com uma maior gratificação profissional, é plausível considerar a maior probabilidade de gralifìcação quando o relacionamento profissional-paciente é bom e 
quando o prognóstico é favorável. Convém destacar, no entanto, a possibilidade de que a motivação de ambas as equipes de saúde não represente a média habitual nos scrviços de saúde, podendo estar muito acima desta.

O componente da avaliação qualitativa denominado de "resultado", portanto, mostrou-se no presente estudo indissociado do componente denominado "processo". As teias que ligam estrutura, processo e resultados são, de fato, bastante frágeis, uma vez que tanto a existência de melhores pré-condições pode ser mal usada quanto a excelência profissional pode resultar em benefícios, mesmo em siluações bastante precárias?. Fica evidente que todo o esforço deveria ser feito no sentido de mellorar a fase de "processo" da assistência primária à saúde, investindo na sua estrutura e, entre outros, na educação continuada de seus profissionais, instrumentalizando-os adequadamente para a clemanda solicitada e para alcançar melhor relação com os pacientes.

Em resumo, o presente estudo revelou que, do ponto de vista dos pacientes, a satisfação com o serviço de saúde está associada com a resolução do problema que motivou a consulta. A disponibilidade de medicamentos no próprio posto de saúde é o principal fator associado com a capacidade resolutiva qualitaliva do serviço. A satisfação do profissional em assistência primária à saúde esteve diretamente associada com a melhor qualidade da relação com o paciente e com uma expectaliva prognóstica mais favorável. Processo e resultados (dois elementos da qualidade dos serviços) estivcram indissociados, indicando que todo o esforço devcria ser feito para melhorar o processo de cuidado dos pacientes.

\section{Agradecimentos}

Às comunidacles e às equipes dos postos de saúde da Vila Santos Dumont e Areal da Cidade de Pelotas, RS. Ao Dr. Fernando Barros do Departamento de Medicina Social da UIPel pelas sugestões e críticas.

HALAL, I.S. et al. [Quality evaluation of primary liealth care an urban area of southern Brazill. Rev. Saitle Puiblica, 28: 131-6, 1994. A 10\% sample of patients who attended two Primary IIcalth Care Units (PHCU) in Pelotas, RS (Brazil) was studied cross-sectinonally for the purpose of measuring qualitatively the attention being offered to the population. According to domiciliar interviews, 15 days after their appointments, a solution (cure or improvenent) was aclicved in $87.9 \%$ of the patients. Patients or parents satisfaction (in the case of pediatric patients) was observed in nearly $90 \%$ of the sample. Palient satisfaction and solution were statistically associated $(p=0.01)$. Association between the solution and the availability of medicines in the I'IICU was observed. Patients who had received the whole or a part of their treatment had $33 \%$ greater probability of solving their problem. Professional satisfaction was lincarly associated with both perception of a better professional-patient relationship $(\mathrm{PR}=3.48 ; \mathrm{CI} 95 \% 2.17-5.59)$ and the expectation of a better patient prognosis (PR=1.99; CI95\%1.36-2.91).

Keytords: Primary health care. Outcome and process assessment (health carc). Consumer satisfaction.

\section{Referências Bibliográficas}

1. BAMIORD, C. \& JACOBY, A. Development of patient satisfaction questionnaires: I. Methodological is sucs. Qual. Assur: Ireallh Care, 1:153-7, 1992.

2. CAMPOS, 1.E. Resolutividade: uma aproximação à avaliação qualitativa dos scrviços de saúde. Rio de Jinciro, 1988. [Tese de Doutorado - Escola Nacional de Saúde Pública/ FIOCRUZ]

3. CENIERS IOR DISEASE CONTROI. Epidemiology Program Oflice. EPI INIO version 5.00. Atlanta, 1990.

4. DONABLDIAN, A. A guide to medical administration. New York, American Public IIcalth Association, 1966.

5. ECCLES, M.; J ACOBY, A.; BAMFORD, C. Development of paticnt satisfaction questionnaires: II. Collaboration in practice. Qual. Assul: Health Care, 1:158$60,1992$.

6. INTIERNATIONAL Classification of IIcalth Problems in Primary Care. ICIIPPC-2-DEFINED. 3th ed. Oxford, WONCA/WIIO/Oxford Mcdical Publication, 1986.

7. MAXWELL, R.J. Dimensions of quality revisited: from trought to action. Qual. Assur. Health Care, 1:1712, 1992.

8. SIPSS/PC++. Statistical package for the social sciences. 2nd cd. New York, McGraw-Hill, 1975.

9. VUORI, II. A qualidade da saúde. Rev. Divulg. Saúde Debale, 3:17-25, 1991.

10. WIII'FIEI.D, M. \& BAKER, R. Measuring patient satisfaction for audit in gencral practice. Qual. Assur. lleallh Care, 1:151.2, 1992.

Recebiclo para publicação em 24.8.1993

Reapresentado em 11.1.1994

Aprovado para publicação em 11.2.1994 